\title{
Epidemiology of Neuromelioidosis in Asia-Pacific: A Systematic Review
}

Mohd 'Ammar Ihsan Ahmad Zamzuri', Mohd Nazrin Jamhari', Haniff Mohd Nawi ${ }^{1}$ (D), Mohd Rohaizat Hassan ${ }^{2}$,

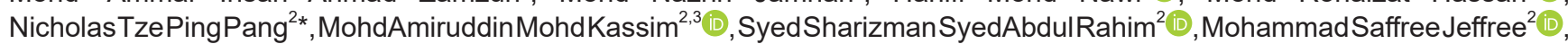
Shi Yun Lee ${ }^{2}$ (D)

${ }^{1}$ Department of Community Health, Faculty of Medicine, Universiti Kebangsaan Malaysia, 56000 Cheras, Kuala Lumpur, Malaysia; ${ }^{2}$ Department of Community and Family Medicine, Faculty of Medicine and Health Sciences, Universiti Malaysia Sabah, 88400 Kota Kinabalu Sabah, Malaysia; ${ }^{3}$ Department of Psychiatry, Faculty of Medicine, Universiti Kebangsaan Malaysia, 56000 Cheras, Kuala Lumpur, Malaysia

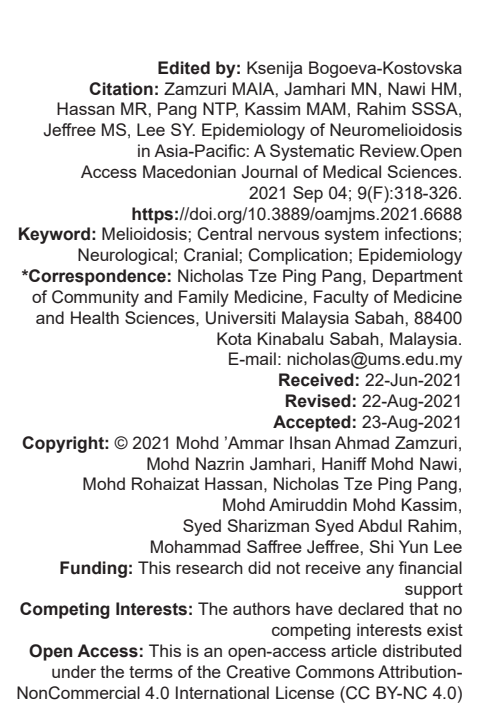

\section{Abstract}

BACKGROUND: Neuromelioidosis is a rare complication of melioidosis caused by Burkholderia pseudomallei, a Gram-negative bacterium commonly found in soil and surface water. Although cerebral involvement of melioidosis comprises only $4 \%$ of total complications, it significantly impacts mortality and morbidity.

AIM: This study aims to perform a systematic review on various neurological complications of melioidosis in the AsiaPacific region within the previous 5 years.

METHOD: Systematic search was performed in PubMed, Web of Science databases and Google Scholar on neuromelioidosis complications published from 2015 to 2019. We excluded animal cases and studies that do not have clinical data.

RESULTS: We identified 283 relevant articles from the initial search, but only 16 articles fulfilled the criteria. Common risk factors identified were diabetes, chronic renal and lung disease, alcohol abuse, and immunosuppression. Neuromelioidosis is commonly detected 6-14 days after the first presentation and confirmed by detailed investigations. Radio imaging investigation helps to differentiate neuromelioidosis from other diagnoses such as meningitis or brain abscess. The majority of literature recommended 3-4-week intensive course of intravenous Ceftazidime or Meropenem therapy, followed by 3-6 months Trimethoprim and Sulfamethoxazole orally for eradication therapy. Oral doxycycline was also prescribed upon discharge. Majority of the patients showed improvement following treatments completion.

CONCLUSION: Neuromelioidosis is rare, with relatively nonspecific central nervous system clinical features. Patients or travelers from endemic areas with risk factors should be treated cautiously. Radio imaging modalities aid early microbiological sampling and appropriate antibiotic therapy.

\section{Introduction}

Melioidosis is caused by Burkholderia pseudomallei, a Gram-negative bacterium commonly encountered in soil and surface water. It is an endemic disease in sub-tropical regions, for instance in Southeast Asia, South Asia, China, and Northern Australia. Incidence generally increases around the wet season [1]. Diabetes, alcohol intake, and chronic kidney disease represent risk factors for developing melioidosis [2]. A wide range of clinical symptoms prevail, which can range from severe multiorgan sepsis to lung symptoms resulting from pulmonary infection, genitourinary, bone, and soft tissue infection [3]. Only $4 \%$ of cases feature cerebral complications; however, the impact on mortality and morbidity is significant [4]. To the best of knowledge, there re no previous systematic reviews in the Asia-Pacific region focusing on epidemiology and associated factors of melioidosis. As the disease reservoir of melioidosis, the Asia-Pacific region has scant resources and health-care facilities; hence, there is an urgent need to allocate resources judiciously using a solid evidence base. The present study aimed to perform a systematic review of the neurological complication of melioidosis among cases in the Asia-Pacific region within the previous 5 years.

\section{Search strategy and selection criteria}

A systematic review was conducted using Preferred Reporting Items for a Systematic Review and Meta-analysis of Individual Participant Data (PRISMAIPD) guideline. The inclusion criteria for the study and case recruitment were as follows: (1) The study must be a case report or case series, (2) all melioidosis cases with diagnosed central nervous system (CNS) complications 
including encephalomyelitis, brain abscess, isolated meningitis, and other confirmed pathology, (3) melioidosis cases required confirmation either by culture, serology, or polymerase chain reaction, and (4) only include studies from the Asia-Pacific region. Exclusion criteria included

(1) animal study, (2) no abstract or full text available, and

(3) the report language was not in English.

Three types of databases were used to find the relevant articles for inclusion which is PubMed database, Web of Science (WOS) database and Google Scholar. The search strategies using MeSH term were as follows:

"melioidosis"

AND

"CNS infections" OR "neurological" OR "cranial"

\section{AND \\ "complication"}

\section{AND}

"epidemiology"

The filters included human studies, English language, and limited to the previous 5 years of publication. The selection of included articles is as described in the PRISMA flow Figure 1. Initially, the literature from all databases yielded was gathered and titles were screened through. Any duplicated articles and unrelated titles deviating from the neurological sequalae of melioidosis were removed during the process. Subsequently, the abstracts were reviewed before removing the unrelated articles. Finally, full texts were reviewed independently by the first three authors for quality checking and information extraction.

\section{Ethics statement}

The article has obtained ethical approval from Medical Research Committee of Faculty of Medicine of a university in Malaysia for this systematic review.

\section{Result}

The search yielded 252 articles from Google Scholar, 23 articles from PubMed and 8 articles from WOS, resulting in 283 unique hits. Only 18 articles were included in the full text assessment after undergoing rigorous selection screening as shown in the PRISMA

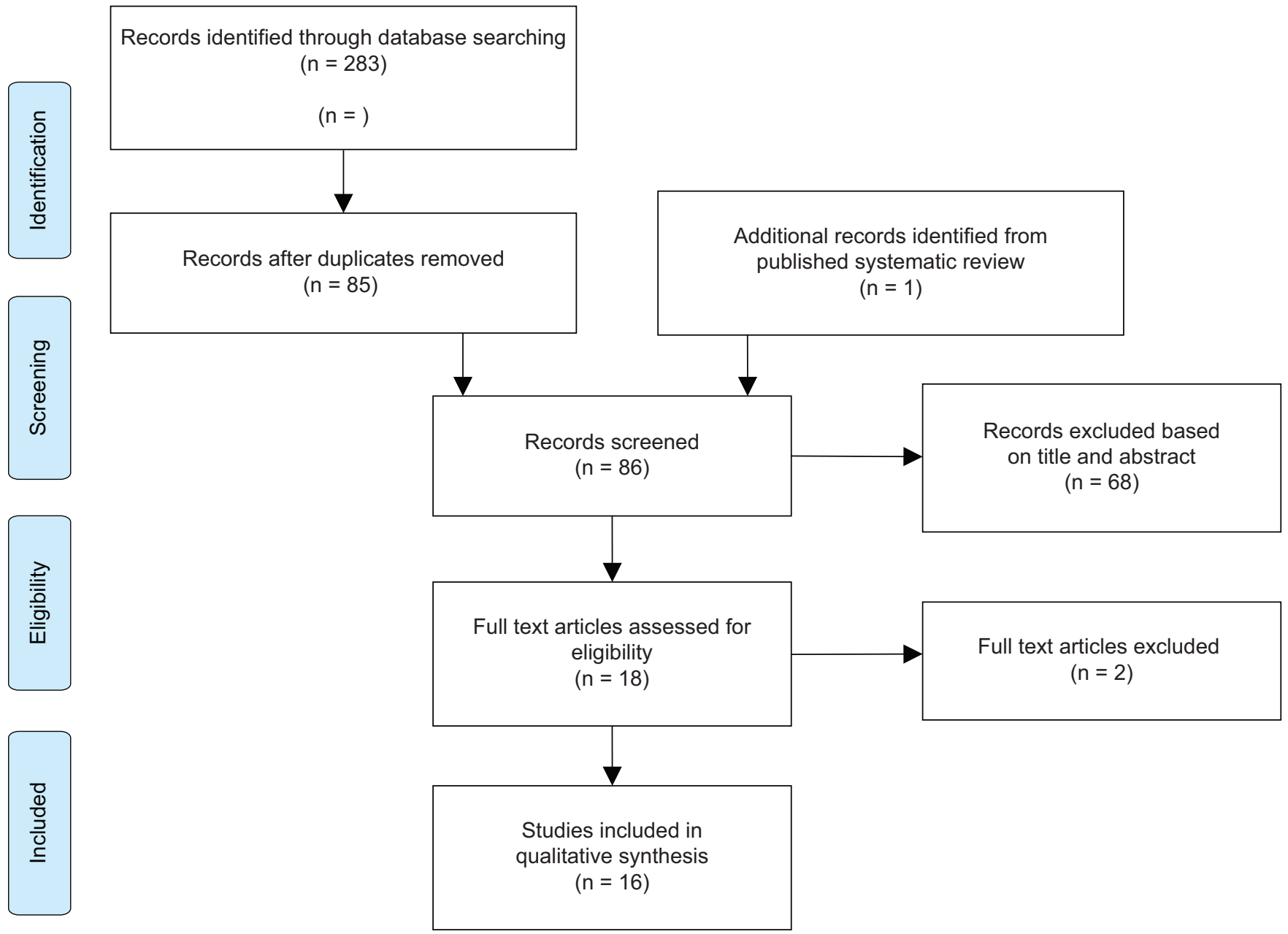

Figure 1: PRISMA flow diagram showing the selection of studies on epidemiology and factor associated of neuromelioidosis in Asia-Pacific 
flow diagram (Figure 1). Ultimately, 16 articles (Table 1, Supplementary Table) were eligible for final systematic review that answered the objectives. Four articles were from India, followed by Australia and Sri Lanka with three articles and two articles each from Malaysia, Taiwan, and China (Figure 2).

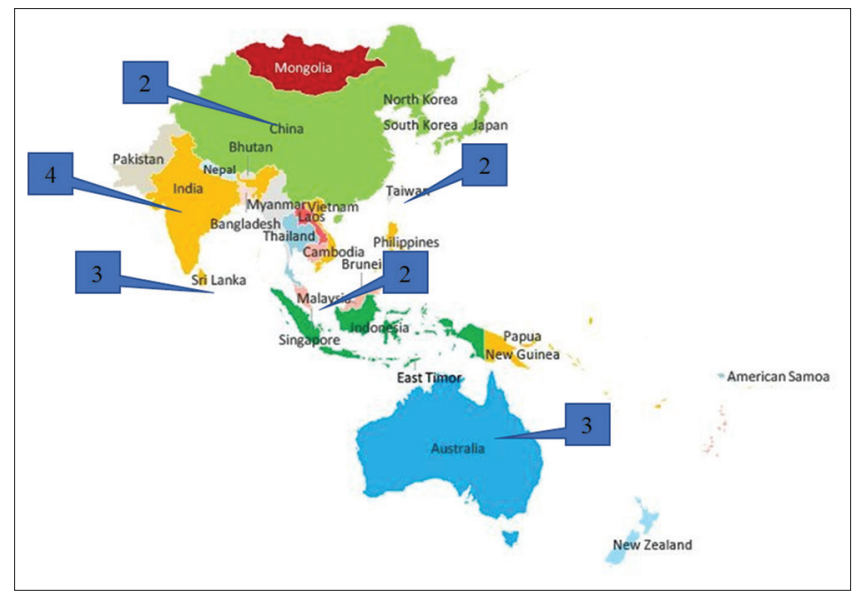

Figure 2: Distribution of articles selected according to country

\section{Characteristic of the study}

Fourteen articles are from case studies describing melioidosis patients who developed complications of neuromelioidosis throughout the treatment period. The remaining articles are retrospective studies and clinical notes discussing neurological complications of melioidosis. Patients who were selected as samples for the case study consisted of 24 males and 11 females, ranging from adult to infants. The age group of the sample ranged from 10 months old to 69 years old.

\section{Risk factor}

The majority of the patients had at least any one comorbid such as diabetes, hypertension, retroviral disease, psychiatric illness, lymphoma, or had a history of smoking and consuming alcohol. Six of the patients were healthy with no comorbidities, while one not specifically mentioned. From the clinical history, all of the patients had exposure to soil and surface water either through travel or agricultural risks. Acquisition of the bacterium occurred potentially through inoculation of contaminated soil. The majority of the cases happened in the rainy season.

\section{Sign and symptoms}

Patients who were selected in this study initially presented with high-grade fever, headache, vomiting, and respiratory symptoms. Most of them were initially diagnosed as pneumonia or fever due to respiratory tract infection and discharged with oral antibiotics and antipyretics. However, most of them had neurological symptoms on re-presentation to casualties. The symptoms commonly reported were seizures, hemiparesis, limb weakness, or delirium.

From the articles selected, the authors observed that the subsequent symptoms were commonly occur within a range of 6-14 days after the first presentation to the hospital. Further investigations were immediately taken to obtain a definitive diagnosis.

\section{Investigations}

Most patients within the current review had raised full blood counts including high leucocyte and neutrophil counts, with no reported abnormalities in other profiles including kidney profiles. Computed tomography scans of the brain and neck and magnetic resonance imaging (MRI) of the brain were performed to narrow down the differential diagnosis as well as to corroborate with the clinical symptoms. Single or multiple ring enhancing lesions was commonly associated with abscess that was the result of melioidosis of the CNS. Subsequently, biopsies and cultures were done to isolate $B$. pseudomallei pathogen, allowing the diagnosis of neuromelioidosis to be made. Radio imaging is hence crucial to distinguish neuromelioidosis from similar diagnoses including meningitis and encephalitis.

\section{Management}

Based on the articles selected, it was observed that treatment of choice for neuromelioidosis trended towards broad spectrum antibiotics. Initial therapy was intravenous course for 3-4 weeks with Meropenem (50 mg/kgupto 2 gevery $8 \mathrm{~h}$ )or Ceftazidime(2gTDS)along with Trimethoprim-Sulfamethoxazole (240/1200 mg (for adults weighing $60 \mathrm{~kg}$ or less)/320/1600 mg (for adults weighing $>60 \mathrm{~kg}$ ) every $12 \mathrm{~h}$ ) followed by 6 months of oral eradication therapy with Trimethoprim-Sulfamethoxazole and Doxycycline.

\section{Quality appraisal}

Overall, the included studies were good in quality. Assessment of quality was done using Joanna Briggs Institute Critical Appraisal Checklist for Case Reports. Each of the study needed to pass the screening questions prior to be chosen for inclusion. Two independent reviewers screened each paper and have agreed $100 \%$ for it to be selected.

\section{Discussion}

Melioidosis falls firmly within the remit of neglected tropical diseases; only around 1300 cases 
Table 1: Table of articles included in the systematic review

\begin{tabular}{|c|c|c|c|c|c|}
\hline Author & Sex/Age & Risk factor & Sign and Symptoms & Investigation & Treatment \\
\hline $\begin{array}{l}\text { Liang et al., } \\
2016 \text { [5] }\end{array}$ & $\mathrm{M} / 30$ & $\begin{array}{l}\text { No underlying co-morbid. } \\
\text { Exposure history: } \\
\text { Recent outdoor survival game } \\
\text { (military simulation) at a grassy and } \\
\text { muddy ground in middle of Taiwan } 1 \\
\text { week before presentation. Claimed } \\
\text { to have bilateral leg abrasion wound } \\
\text { after the activity. }\end{array}$ & $\begin{array}{l}\text { On presentation: } \\
\text { 1. Acute left (unilateral) limb weakness. Power of } \\
\text { left upper limb (1/5) while left lower limb }(2 / 5) \\
\text { 2. Partial seizures (seconds) Otherwise afebrile, } \\
\text { normal vital sign and all limbs' reflexes were } \\
\text { normal. } \\
\text { Progress: } \\
\text { Spiking temperature and stupor (GCS } 7 \text {, } \\
\text { E2V1M4) } 3 \text { days later. }\end{array}$ & $\begin{array}{l}\text { Confirmed by Blood and Pus culture } \\
\text { Imaging (during febrile and stupor) } \\
\text { CT brain - swelling in the right } \\
\text { hemisphere with midline shift } \\
\text { MRI - abscess in the right frontal- } \\
\text { parietal lobe, with extension to } \\
\text { thalamus, cerebral peduncle, and } \\
\text { medial temporal lobe, crossing } \\
\text { the midline via corpus callosum to } \\
\text { the subependymal area of the left } \\
\text { lateral ventricle. }\end{array}$ & $\begin{array}{l}\text { 1. IV Meropenem } 2 \mathrm{~g} 8 \mathrm{~h} \text { for } \\
12 \text { weeks followed } \\
\text { 2. T. Trimethoprim- } \\
\text { sulfamethoxazole for } 3 \\
\text { months } \\
\text { 3. Intraventricular } \\
\text { Ceftazidime for } 2 \text { weeks } \\
\text { (EVD) }\end{array}$ \\
\hline $\begin{array}{l}\text { M. White et al., } \\
2016 \text { [6] }\end{array}$ & $\mathrm{M} / 14$ & $\begin{array}{l}\text { 1. Aboriginal population } \\
\text { 2. History of solvent abuse }\end{array}$ & $\begin{array}{l}\text { 1. Ataxia } \\
\text { 2. Severe headache } \\
\text { 3. Vomiting } \\
\text { 4. Unilateral (left) facial and limb weakness } \\
\text { 5. Dysphasia }\end{array}$ & $\begin{array}{l}\text { Confirmed by biopsy of the } \\
\text { cerebellar mass. } \\
\text { MRI: Leptomeningeal } \\
\text { enhancement, ring-enhancing } \\
\text { lesions, edema, abscess. }\end{array}$ & $\begin{array}{l}\text { 1. IV Meropenem } \\
\text { 2. Oral Cotrimoxazole }\end{array}$ \\
\hline $\begin{array}{l}\mathrm{Ng} \text { et al., } \\
2015 \text { [7] }\end{array}$ & $\mathrm{M} / 62$ & $\begin{array}{l}\text { 1. Type } 2 \text { Diabetes } \\
\text { 2. Hypertension } \\
\text { 3. Work as local council truck driver } \\
\text { transporting water to local garden } \\
\text { and public area. }\end{array}$ & $\begin{array}{l}\text { On presentation: } \\
\text { 1. Fever } \\
\text { 2. Vomiting } \\
\text { 3. Fatigue } \\
\text { Progression: Developed parkinsonism after } \\
\text { treatment (day } 7 \text { admission) } \\
\text { 1. Generalized rigidity of neck, trunk and limb } \\
\text { 2. Mask like facies } \\
\text { 3. Bradykinesia } \\
\text { 4. Bradyphrenia with monotonous speech } \\
\text { 5. Bilateral resting tremor both hands }\end{array}$ & $\begin{array}{l}\text { Confirmed by blood culture. } \\
\text { Laboratory: Elevated WBC } \\
\text { predominant neutrophils, } \\
\text { hyponatremia ( } 115 \mathrm{mmol} / \mathrm{L}) \\
\text { MRI and CT scan - noted brain } \\
\text { was normal }\end{array}$ & $\begin{array}{l}\text { 1. IV Imipenem } \\
\text { 2. Oral Cotrimoxazole } \\
\text { 3. Oral Doxycycline }\end{array}$ \\
\hline $\begin{array}{l}\text { Wijekoon et al., } \\
2016 \text { [8] }\end{array}$ & $F / 42$ & 1. Type 2 Diabetes & $\begin{array}{l}\text { On presentation: } \\
\text { 1. Recurrent skin and liver abscess } \\
\text { 2. Fever (intermittent) } \\
\text { 3. Abdominal pain } \\
\text { 4. Vomiting } \\
\text { Progression: Subsequently developed GBS } \\
\text { after treatment (day } 7 \text { admission) } \\
\text { 1. Bilateral lower limb weakness }\end{array}$ & $\begin{array}{l}\text { Confirmed by PCR and inhouse } \\
\text { indirect hemagglutination (IHA) } \\
\text { Laboratory: Elevated WBC with } \\
\text { granulocytosis }\end{array}$ & $\begin{array}{l}\text { 1. IV Meropenem } \\
\text { 2. Oral Cotrimoxazole } \\
\text { 3. Oral Co-amoxiclav }\end{array}$ \\
\hline $\begin{array}{l}\text { Chen et al., } \\
2018 \text { [9] }\end{array}$ & $\mathrm{F} / 65$ & $\begin{array}{l}\text { Anorexia } \\
\text { Depressive disorder. }\end{array}$ & $\begin{array}{l}\text { 1. Poor appetite and fatigue } \\
\text { 2. Confusion } \\
\text { 3. Acute fever } \\
\text { 4. Headache } \\
\text { 5. Lead-pipe rigidity of all limbs } \\
\text { 6. Delirium }\end{array}$ & $\begin{array}{l}\text { Confirmed by CSF culture. } \\
\text { Laboratory: Raised WBC }\end{array}$ & $\begin{array}{l}\text { 1. IV Ceftazidime } 2 g \text { TDS for } \\
3 \text { weeks } \\
\text { 2. Oral Cotrimoxazole } \\
\text { 3. Oral Minocycline }\end{array}$ \\
\hline $\begin{array}{l}\text { Prasanna Kumar } \\
\text { et al., } 2017 \text { [10] }\end{array}$ & $\mathrm{F} / 10 \mathrm{~m}$ & $\begin{array}{l}\text { Exposure to wet soil in a pond } \\
\text { adjacent to the house during rainy } \\
\text { season. } \\
\text { Born from 3rd degree } \\
\text { consanguineous parents }\end{array}$ & $\begin{array}{l}\text { On presentation: } \\
\text { 1. High grade fever ( } 2 \text { weeks) } \\
\text { 2. Multiple subcutaneous swelling } \\
\text { 3. Cough ( } 1 \text { week) } \\
\text { Progression: Developed CNS symptoms after } \\
\text { treatment (day } 6 \text { of admission) } \\
\text { 1. Irritability } \\
\text { 2. Left eye medial rectus palsy } \\
\text { 3. Plantar reflex - flexor (down going) }\end{array}$ & $\begin{array}{l}\text { Confirmed by cultures from the } \\
\text { nodule aspirate } \\
\text { Laboratory: elevated WBC } \\
\text { MRI: mid-brain and pontine patchy } \\
\text { nodular enhancing foci. }\end{array}$ & $\begin{array}{l}\text { 1. IV Meropenem for } 6 \\
\text { weeks } \\
\text { 2. Oral Cotrimoxazole }\end{array}$ \\
\hline $\begin{array}{l}\text { Arif et al., } \\
2015 \text { [11] }\end{array}$ & $\mathrm{M} / 33$ & $\begin{array}{l}\text { No underlying co-morbid. } \\
\text { Work as a zoo worker, took care of } \\
\text { primarily healthy lions and tiger. } \\
\text { Exposure history: } \\
\text { Has a travel history of a recently to } \\
\text { Sri Lanka }\end{array}$ & $\begin{array}{l}\text { On presentation: } \\
\text { 1. } 3 \text {-day history of nausea, vomiting, and } \\
\text { difficult walking. } \\
\text { 2. Clinically diaphoretic, afebrile, BP and heart } \\
\text { rate stable. Tachypneic at } 18 \text { breaths/min. No } \\
\text { dysautonomia. } \\
\text { 3. Neurological examination: Flaccid } \\
\text { quadriparesis with } 3 / 5 \text { power in both upper } \\
\text { and lower extremities, absent reflexes. } \\
\text { Progress: } \\
\text { 1. Dysphagia } \\
\text { 2. Loss of protective reflex that required } \\
\text { mechanical ventilation. } \\
\text { 3. Further deterioration - consciousness level } \\
\text { worsened; bilateral pinpoint pupils with } \\
\text { absent corneal reflexes. } \\
\text { 4. Gaze paresis in horizontal directions } \\
\text { bilaterally with pre- served vertical gaze on } \\
\text { oculocephalic reflex testing. } \\
\text { 5. Motor power reduced } 1 / 5 \text { in upper and lower } \\
\text { limbs. All deep tendon reflexes were absent } \\
\text { and plantars were flexor. }\end{array}$ & $\begin{array}{l}\text { Confirmed by: Bronchoalveolar } \\
\text { culture grew B. pseudomallei. } \\
\text { Bronchoscopy was done when } \\
\text { repeated (2-week) chest x-ray } \\
\text { showed cavitation at left mid zone } \\
\text { and CT-thorax showed bilateral } \\
\text { lower lobe consolidation, with left } \\
\text { lobe cavity lesion. } \\
\text { CSF examination: Lymphocytic } \\
\text { pleocytosis with raised protein level } \\
\text { but normal glucose concentration. } \\
\text { MRI Brain (with gadolinium): } \\
\text { multiple small abscesses with ring } \\
\text { enhancement at posteroinferior } \\
\text { brainstem and upper cervical cord. } \\
\text { Repeated MRI following } \\
\text { deterioration showed worsening } \\
\text { and extension - involving bilateral } \\
\text { thalamus, internal capsule } \\
\text { extending up to the corona radiata } \\
\text { along the corticospinal tract, } \\
\text { medulla oblongata and cerebellum. }\end{array}$ & $\begin{array}{l}\text { 1. Cotrimoxazole for } 4 \\
\text { weeks, and } \\
\text { 2. Meropenem for } 4 \text { weeks }\end{array}$ \\
\hline $\begin{array}{l}\text { Loh et al., } \\
2017 \text { [12] }\end{array}$ & $M / 35$ & $\begin{array}{l}\text { No underlying co-morbid. } \\
\text { Exposure history: } \\
\text { History of pig-hunting barefooted. } \\
\text { Lying on the bare ground for } \\
\text { prolonged periods (to fix a car) } 2 \\
\text { weeks prior to presentation }\end{array}$ & $\begin{array}{l}\text { On presentation: } \\
\text { 1. 3-day history of right temporal facial pain } \\
\text { radiating to the temporomandibular joint } \\
\text { (exacerbated by chewing). } \\
\text { 2. Night sweats and chills } \\
\text { Progress: } \\
\text { 1. Trismus, right parotid and temporal swelling, } \\
\text { fevers, and acute confusion. } \\
\text { 2. Type } 1 \text { respiratory failure and vasodilatory } \\
\text { failure. }\end{array}$ & $\begin{array}{l}\text { Confirmed by: Blood cultures. } \\
\text { Blood investigations: } \\
\text { Leukocytosis, raised CRP, and acute } \\
\text { kidney injury (creatinine } 241 \text { mmol/L) } \\
\text { CT (head and neck): Inflamed right } \\
\text { parotid and soft tissue. Complete } \\
\text { opacification of the right middle ear } \\
\text { and mastoid cavity without overt } \\
\text { bony destruction. Low density focus } \\
\text { with no contrast enhancement } \\
\text { suggestive of cerebritis. }\end{array}$ & $\begin{array}{l}\text { 1. IV Meropenem for } 4 \\
\text { weeks } \\
\text { 2. IV Ceftazidime for } 4 \\
\text { weeks } \\
\text { 3. Oral Cotrimoxazole } \\
\text { therapy for } 6 \text { months }\end{array}$ \\
\hline
\end{tabular}


Table 1: (Continued)

\begin{tabular}{|c|c|c|c|c|c|}
\hline Author & Sex/Age & Risk factor & Sign and Symptoms & Investigation & Treatment \\
\hline $\begin{array}{l}\text { Hsu et al., } \\
2016 \text { [13] }\end{array}$ & $\begin{array}{l}\text { Cases: } \\
\text { Patient 1: } \\
\text { M/43 } \\
\text { Patient 2: } \\
\text { F/69 } \\
\text { Patient 3: } \\
\text { M/56 } \\
\text { Patient 4: } \\
\text { M/41 } \\
\text { Patient 5: } \\
\text { F/14 } \\
\text { Patient 6: } \\
\text { F/13 } \\
\text { Patient 7: } \\
\text { F/23 } \\
\text { Patient 8: } \\
\text { F/62 } \\
\text { Patient 9: } \\
\text { F/43 } \\
\text { Patient 10: } \\
\text { M/52 }\end{array}$ & $\begin{array}{l}\text { Identifiable risk factors: } \\
\text { 1. Diabetes } \\
\text { 2. Immunosuppression } \\
\text { 3. Alcohol abuse } \\
\text { 4. Outdoor occupation }\end{array}$ & $\begin{array}{l}\text { Reviewed symptoms of the cases: } \\
\text { 1. Fever }(n=9) \\
\text { 2. Headache }(n=9) \\
\text { 3. Neck stiffness }(n=2) \\
\text { 4. Photophobia }(n=5) \\
\text { 5. Ipsilateral trigeminal nerve paresthesia in } \\
\text { the maxillary distribution }(n=3) \\
\text { 6. Bilateral lower limb paresthesia }(n=1) \\
\text { 7. Profound expressive aphasia }(n=1)\end{array}$ & $\begin{array}{l}\text { Cases were confirmed by cultures: } \\
\text { 1. Blood culture: Patient } 1 \text { and } \\
\text { Patient } 7 \\
\text { 2. CSF culture: Patient } 6 \text { and } \\
\text { Patient } 8 \\
\text { 3. Extradural culture: Patient } 9 \\
\text { and Patient } 10 \\
\text { 4. Brain biopsy: Patient } 5 \text { and } \\
\text { Patient } 2 \\
\text { 5. Respiratory aspirate: Patient } 3 \\
\text { and Patient } 4 \\
\text { MRI brain/spine: } \\
\text { - Seven patients - demonstrated } \\
\text { rim-enhancing micro abscesses } \\
\text { with propensity for white matter } \\
\text { tracts including the corticospinal } \\
\text { tracts, corpus callosum, and } \\
\text { cerebellar peduncles. } \\
\text { - Three of these } 7 \text { patients } \\
\text { also showed thickening and } \\
\text { enhancement of the trigeminal } \\
\text { nerves with contiguous spread } \\
\text { to brain stem trigeminal nuclei. } \\
\text { - Three patients had isolated } \\
\text { extra axial disease with } \\
\text { findings including meningeal } \\
\text { enhancement, extradural } \\
\text { abscess, skull osteomyelitis, } \\
\text { and scalp abscess }\end{array}$ & Not specifically mentioned \\
\hline $\begin{array}{l}\text { Huang et al., } \\
2018 \text { [14] }\end{array}$ & $\mathrm{M} / 38$ & $\begin{array}{l}\text { 1. Type } 2 \text { Diabetes } \\
\text { 2. } 10 \text {-years smoker }\end{array}$ & $\begin{array}{l}\text { On presentation: } \\
\text { 1. Cough and blood-tinge expectorate. } \\
\text { Progress: (after } 8 \text { months) } \\
\text { 1. Acute left limb weakness, hemiopia, } \\
\text { seizure, and headache. }\end{array}$ & 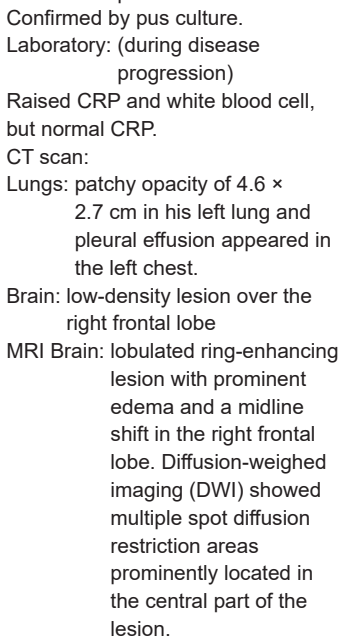 & $\begin{array}{l}\text { 1. IV Imipenem/Cilastin for } \\
6 \text { weeks } \\
\text { 2. IV Doxycycline for } 6 \\
\text { weeks } \\
\text { 3. Oral Doxycycline for } 4 \\
\text { months } \\
\text { 4. Oral Cotrimoxazole for } 4 \\
\text { months }\end{array}$ \\
\hline $\begin{array}{l}\text { Amarasena } \\
\text { et al., } 2019 \text { [15] }\end{array}$ & $\mathrm{M} / 42$ & $\begin{array}{l}\text { 1. Type } 2 \text { Diabetes } \\
2 \text {. Work as a grocer }\end{array}$ & $\begin{array}{l}\text { On presentation } \\
\text { 1. Fever for } 6 \text { days } \\
\text { 2. Headache } \\
\text { 3. constitutional symptoms } \\
\text { Progression: } \\
\text { - Simple partial seizures involving the left lower } \\
\text { limb, progressing to persistent numbness of } \\
\text { the left side of the body }\end{array}$ & $\begin{array}{l}\text { Diagnosis established by indirect } \\
\text { hemagglutination assay (IHA) for } \\
\text { melioidosis antibody (antibody titer } \\
\text { of more than } 1 / 10,240 \text { ) } \\
\text { CT scan (head): subdural collection } \\
\text { over the right fronto-parietal region } \\
\text { with gas locules and obliteration } \\
\text { of sulci and gyri, without definite } \\
\text { evidence of abscess formation. } \\
\text { MRI (contrast) of the brain: a } \\
\text { subdural collection in the right } \\
\text { fronto-temporo-parietal region with } \\
\text { possible abscess formation in the } \\
\text { right parietal region. }\end{array}$ & $\begin{array}{l}\text { 1. IV Meropenem for } 8 \\
\text { weeks } \\
\text { 2. Oral Cotrimoxazole for } 8 \\
\text { weeks then to continue for } \\
6 \text { months } \\
\text { 3. Oral Doxycycline for } 6 \\
\text { months }\end{array}$ \\
\hline $\begin{array}{l}\text { Zhan et al., } \\
2017 \text { [16] }\end{array}$ & $\begin{array}{l}\text { Cases: } \\
\text { Patient 1: } \\
\text { M/31 } \\
\text { Patient 2: } \\
\text { M/48 } \\
\text { Patient 3: } \\
\text { M/56 } \\
\text { Patient 4: } \\
\text { M/65 } \\
\text { Patient 5: } \\
\text { M/9 } \\
\text { Patient 6: } \\
\text { M/60 } \\
\text { Patient 7: } \\
\text { M/66 }\end{array}$ & $\begin{array}{l}\text { Identifiable risk factors: } \\
\text { 1. Diabetes mellitus } \\
\text { 2. Smoker } \\
\text { 3. Working in agriculture }\end{array}$ & $\begin{array}{l}\text { Reviewed symptoms of the cases: } \\
\text { 1. High fever }(n=5) \\
\text { 2. Headache }(n=4) \\
\text { 3. Chronic chest pain }(n=1) \\
\text { 4. Dizziness }(n=1) \\
\text { 5. Limb weakness }(n=1)\end{array}$ & $\begin{array}{l}\text { Cases were confirmed by cultures: } \\
\text { 1. Blood culture: Patient } 1, \text { Patient } \\
\text { 2. Patient } 3 \text {, Patient } 5 \text {, Patient } 6 \text {, } \\
\text { Patient } 7 \text {. } \\
\text { 2. Culture from brain abscess: } \\
\text { Patient } 4 \\
\text { Laboratory: raised Peripheral blood } \\
\text { leukocytes and the } \\
\text { neutrophil ratio in all } \\
\text { patients. } \\
\text { - CT scan/MRI: mainly manifested } \\
\text { as intracerebral } \\
\text { single or multiple } \\
\text { nodules, as well as } \\
\text { ring and flake-like } \\
\text { enhancements } \\
\text { with rapid lesion } \\
\text { progression }\end{array}$ & Not specifically mentioned. \\
\hline
\end{tabular}


Table 1: (Continued)

\begin{tabular}{|c|c|c|c|c|c|}
\hline Author & Sex/Age & Risk factor & Sign and Symptoms & Investigation & Treatment \\
\hline $\begin{array}{l}\text { Ganapathy } \\
\text { et al., } 2018 \text { [17] }\end{array}$ & $\begin{array}{l}\text { Cases: } \\
\text { Patient 1: } \\
\text { M/39 } \\
\text { Patient2: } \\
\text { M/41 }\end{array}$ & $\begin{array}{l}\text { 1. Type } 2 \text { Diabetes (Patient 1) } \\
\text { 2. Pathological fracture of the } \\
\text { radius, secondary to melioidosis } \\
\text { (Patient } 1 \text { ) } \\
\text { 3. Retroviral disease (Patient 2) }\end{array}$ & $\begin{array}{l}\text { On presentation: } \\
\text { Patient } 1 \\
\text { 1. Head swelling } \\
\text { 2. Spontaneous "purulent" discharge at the } \\
\quad \text { swelling site. } \\
\text { Patient } 2 \\
\text { 1. Fever } \\
\text { 2. Head swelling }\end{array}$ & $\begin{array}{l}\text { Confirmed by pus cultures. } \\
\text { CT scan: } \\
\text { Patient 1: Ring enhancing } \\
\text { iso-hypodense lesion } \\
\text { suggestive of intra- } \\
\text { parenchymal abscess. } \\
\text { Patient } 2 \text { (contrast): Subdural } \\
\quad \text { collection. } \\
\text { MRI (Patient 1): T2 hyper intense, } \\
\text { diffusion restricted } \\
\text { collection. }\end{array}$ & $\begin{array}{l}\text { Patient 1: } \\
\text { 1. IV Ceftazidime for } 2 \\
\text { weeks } \\
\text { 2. Oral Doxycycline for } 3 \\
\text { months } \\
\text { Patient 2: } \\
\text { 1. IV Ceftazidime for } 8 \\
\text { weeks }\end{array}$ \\
\hline $\begin{array}{l}\text { Saravu et al., } \\
2015[18]\end{array}$ & $\begin{array}{l}\text { Cases: } \\
\text { Patient 1: } \\
\text { M/39 } \\
\text { Patient 2: } \\
\text { M/45 }\end{array}$ & $\begin{array}{l}\text { Patient 1: } \\
\text { 1. Alcoholic } \\
\text { 2. Type } 2 \text { Diabetes (uncontrol) } \\
\text { Patient 2: } \\
\text { 1. Work as manual laborer }\end{array}$ & $\begin{array}{l}\text { On presentation: } \\
\text { Patient } 1 \\
\text { 1. Fever } \\
\text { 2. Cough } \\
\text { 3. Focal seizures with secondary } \\
\text { generalization. } \\
\text { Patient } 2 \\
\text { 1. Left lower limb weakness } \\
\text { 2. Difficulty in passing urine and stools } \\
\text { 3. Chronic lower back pain }\end{array}$ & $\begin{array}{l}\text { Confirmed by cultures: } \\
\text { 1. Blood culture: Patient } 1 \\
\text { 2. CSF culture: Patient } 2 \\
\text { MRI } \\
\text { Patient 1: small peripherally } \\
\text { enhancing subdural } \\
\text { collection along the } \\
\text { interhemispheric fissure } \\
\text { suggestive of minimal } \\
\text { subdural empyema. } \\
\text { Patient 2: T2 hyperintensity } \\
\text { from the level of D9 } \\
\text { to L1 suggestive of } \\
\text { demyelination }\end{array}$ & $\begin{array}{l}\text { Patient 1: } \\
\text { 1. IV Ceftazidime } 2 \mathrm{~g} \text { QID } \\
\text { for } 2 \text { weeks } \\
\text { 2. Oral Cotrimoxazole } 320 \\
\mathrm{mg} / 1600 \mathrm{mg}, \mathrm{BD} \text { for } 6 \\
\text { months } \\
\text { 3. Oral Doxycycline } 100 \\
\text { mg BD for } 6 \text { months } \\
\text { Patient } 2 \text { : } \\
\text { 1. IV Meropenem } 1 \mathrm{~g} \text { TDS } \\
\text { for } 3 \text { weeks } \\
\text { 2. Oral Cotrimoxazole } 320 \\
\mathrm{mg} / 1600 \mathrm{mg}, \mathrm{BD} \text { for } 3 \\
\text { weeks } \\
\text { 3. Oral Doxycycline } \\
\text { 100mg BD for } 6 \text { months }\end{array}$ \\
\hline $\begin{array}{l}\text { Fong et al., } \\
2017[19]\end{array}$ & $\begin{array}{l}\text { Cases: } \\
\text { Patient 1: } \\
\text { F/47 } \\
\text { Patient 2: } \\
\text { M/16 } \\
\text { Patient 3: } \\
\text { F/67 }\end{array}$ & $\begin{array}{l}\text { Patient 1: } \\
\text { 1. Smoker } \\
\text { 2. No underlying co-morbid } \\
\text { Patient 2: } \\
\text { 1. Lymphoma } \\
\text { Patient 3: } \\
\text { 1. Hypertension } \\
\text { 2. Type } 2 \text { Diabetes }\end{array}$ & $\begin{array}{l}\text { On presentation: } \\
\text { Patient 1: } \\
\text { 1. Fever } \\
\text { 2. Generalized headache } \\
\text { 3. Lethargy } \\
\text { 4. Frontal scalp swelling } \\
\text { Patient 2: } \\
\text { 1. Fever } \\
\text { 2. Vomiting } \\
\text { 3. Diarrhea } \\
\text { 4. Generalized tonic-clonic seizure } \\
\text { Patient 3: } \\
\text { 1. Fever } \\
\text { 2. Cough }\end{array}$ & $\begin{array}{l}\text { Confirmed by cultures: } \\
\text { 1. Pus culture: Patient } 1 \\
\text { 2. Blood culture: Patient } 2 \text { and } \\
\text { Patient } 3 \\
\text { MRI } \\
\text { Patient 1: a mass at mid frontal } \\
\text { region which extended } \\
\text { inferiorly and eroded the } \\
\text { left frontal bone. } \\
\text { Patient 2: multiple abscesses of } \\
\text { various sizes in the left } \\
\text { temporal regions with } \\
\text { surrounding white matter } \\
\text { edema. } \\
\text { Patient 3: two large enhancing } \\
\text { lesions within the } \\
\text { right parietal lobe with } \\
\text { perilesional edema, } \\
\text { meningeal enhancement, } \\
\text { adjacent subdural } \\
\text { collection, and an } \\
\text { overlying scalp abscess } \\
\text { with periosteum erosion. }\end{array}$ & $\begin{array}{l}\text { Patient 1: } \\
\text { 1. IV Meropenem 2g TDS } \\
\text { for } 2 \text { weeks } \\
\text { 2. IV Ceftazidime } 2 \mathrm{G} \text { QID } \\
\text { for } 4 \text { weeks } \\
\text { 3. Oral Cotrimoxazole } \\
\text { (TMP- SMX) 240/1200 } \\
\text { mg BD for } 1 \text { year } \\
\text { Patient 2: } \\
\text { 1. IV Meropenem 2g TDS } \\
\text { for } 2 \text { weeks } \\
\text { 2. Oral Cotrimoxazole } \\
\text { 320/1600 mg BD for } 6 \\
\text { months } \\
\text { Patient } 3 \text { : } \\
\text { 1. IV Meropenem } 2 \mathrm{~g} \text { TDS } \\
\text { for } 10 \text { days } \\
\text { 2. IV Ceftazidime } 2 \mathrm{~g} \text { QID } \\
\text { for } 4 \text { weeks } \\
\text { 3. Oral Cotrimoxazole } \\
\text { 320/1600 mg BD for } 6 \\
\text { months }\end{array}$ \\
\hline $\begin{array}{l}\text { Nayak et al., } \\
2018[20]\end{array}$ & $\mathrm{M} / 23$ & Not mentioned & $\begin{array}{l}\text { 1. Progressive diplopia (looking towards the left). } \\
\text { 3. Progressive decrease hearing on left ear. }\end{array}$ & $\begin{array}{l}\text { Confirmed by biopsy culture. } \\
\text { MRI of the brain with contrast } \\
\text { showed pachymeningeal thickening } \\
\text { and abnormal enhancement along } \\
\text { the left temporal region, the petrous } \\
\text { apex, and within the left mastoid } \\
\text { air cells. } \\
\text { Magnetic resonance venogram } \\
\text { (MRV) showed chronic partial } \\
\text { thrombosis of the left transverse- } \\
\text { sigmoid sinus and left jugular vein. }\end{array}$ & $\begin{array}{l}\text { 1. IV Ceftazidime for } 6 \text { weeks } \\
\text { 2. Oral Cotrimoxazole for } 6 \\
\text { months }\end{array}$ \\
\hline
\end{tabular}

have been reported since 2010 [21]. This is undoubtedly a gross underreporting, based on a far higher projected incidence based on a statistical model in 2016 [22]. This could be correlated with prolonged latency periods; it usually ranges from 1 to 21 days, but there have been instances where latent periods were delayed for months or years [23]. Another factor contributing to underreporting is the multiplicity of potential presentations. This is because it can present as a limited infection of one body system, or as a disseminated systemic infection. Likewise, sterile cultures may negatively rule out the important diagnosis and left patient untreated [24]. Pneumonia is the most common presentation, accounting for approximately half of all presentations. Other infective presentations include but are not limited to prostatic abscesses, parotitis, skin and soft tissue infections, and encephalomyelitis. These presentations are not entirely independent but can be related to various predisposing factors including diabetes mellitus, chronic renal failure, malignant tumors, alcohol abuse, liver disease, steroid use, and lymphoid or myeloid disorders. This is corroborated in the Asia-Pacific region through this systematic review, where many younger clients had complications of diabetes mellitus. Hence, it could be a significant risk factor in this particular age group [6], [8], [13].

Despite CNS infections comprising only $5 \%$ of all melioidosis cases, reporting has expanded tremendously in the past few years [12]. This can be attributed both to better disease awareness as well 
as better laboratory capacity [25]. CNS presentations include headaches, altered level of consciousness, seizures, cerebellar signs, cranial nerve palsies, limb weakness, and hemiparesis [26], [27]. With a one-quarter mortality rate and significant survivor morbidity, early recognition is crucial for better prognosis [10], [13], [28].

MRI features of CNS melioidosis include rim enhancing cerebral microabscesses, which may be rounded or tubular in morphology. Nevertheless, other CNS infections can present with similar microabscess morphology including toxoplasmosis, neurocysticercosis, and tuberculosis [15], [16]. However, the propensity to spread along and involve white matter tracts across the commissural and projection fibers to the contralateral hemisphere is another distinguishing feature of neuromelioidosis. This commissural fiber spread may hence explain bilateral but asymmetric distributions of melioidosis lesions, favoring the hemisphere of initial infection. Supra and infratentorial structures as well as the spinal cord are involved in this dissemination, which results in difficulties in organism eradication and consequent poor clinical outcomes [18], [29].

However, it is often difficult to differentiate melioidosis from brain malignancy using conventional imaging; diffusion-weighted MRI (DWI) can instead increase confidence in distinguishing abscesses from cystic tumors [14]. The criteria for pyogenic abscesses are diffuse hyperintensities on DWI, whereas the criteria for cystic tumors are hypointensity. Using such criteria thus results in $95 \%$ accuracy in diagnosis. Nevertheless, exceptions can still occur; instead of typical diffusion restriction of the brain abscess, one of the patients demonstrated sporophytic high signals on DWI. A possible explanation could be that the early stage of the brain abscess resulted in localized inflammation and abscess cavity formation. However, this case presented as an early abscess that was misdiagnosed as a malignancy. Therefore, the possibility of abscess should be primarily considered when DWI shows diffuse hyperintensity or spot hyperintensity [5], [9].

Understanding the pathogenesis of the disease will aids the clinician to come to the diagnosis. Neuromelioidosis appears to have a variable pathophysiology. To begin, hematogenous dissemination of the bacterium to the CNS is critical in the process. Blood is the most often isolated specimen of $B$. pseudomallei and the circulating microorganisms has the potential to by-pass cellular boundaries, including the blood-brain and blood-cerebrospinal fluid barriers. Few mechanisms were proposed such as through transcellular, paracellular, or Trojan horse [30]. Nevertheless, direct brainstem invasion through the nasal route and cranial nerves is believed to be another distinct pathway of CNS melioidosis, particularly that cause encephalomyelitis [31] as demonstrated in Darwin's study [32]. Apart from that, percutaneous inoculation at scalp area could possibly cause direction extension to the skull and the brain in some cases. This is corroborated in a paper that showed a quarter of the cases reported for CNS melioidosis had contemporaneous skull osteomyelitis or scalp abscess with the pervious history of cranial injuries [33].

A high index of suspicion, early diagnosis, and early initiation of high doses of appropriate antibiotics for a long duration are crucial, as mortality rates in infected individuals are otherwise very high [7], [17]. Clinical presentations, imaging characteristics, and histopathology of melioidosis can mimic tuberculosis, leading to inappropriate treatment with antituberculosis therapy. Current guidelines for the treatment of melioidosis recommend a 2-week intensive therapy with either ceftazidime or meropenem, followed by oral eradication therapy using trimethoprim and sulfamethoxazole for 3-6 months. There is some evidence that suggest better outcomes with meropenem in severe melioidosis with septic shock [14], [20].

\section{Conclusion}

CNS melioidosis is a rare and relatively nonspecific presentation. Coupled with risk factors, MRI offers best diagnostic clue with rim-enhancing tubular micro abscesses affecting the white matter tracts, cranial nerve nuclei, and the cranial nerves. In endemic areas and in returned travelers from endemic regions, the characteristic imaging features should alert the clinician of the possible diagnosis of neuromelioidosis. This will help to direct early microbiological sampling and immediately instituted appropriate antibiotic therapy.

\section{References}

1. Yip TW, Hewagama S, Mayo M, Price EP, Sarovich DS, Bastian I, et al. Endemic melioidosis in residents of desert region after atypically intense rainfall in central Australia, 2011. Emerg Infect Dis. 2015;21(6):1038-40. https://doi.org/10.3201/ eid2106.141908

PMid:25988301

2. Currie BJ, Jacups SP, Cheng AC, Fisher DA, Anstey NM, Huffam SE, et al. Melioidosis epidemiology and risk factors from a prospective whole-population study in northern Australia. Trop Med Int Health. 2004;9(11):1167-74. https://doi. org/10.1111/j.1365-3156.2004.01328.x

PMid:15548312

3. Currie BJ, Fisher DA, Howard DM, Burrow JN, Lo D, SelvaNayagam S, et al. Endemic melioidosis in Tropical Northern Australia: A 10-year prospective study and review of the literature. Clin Infect Dis 2000;31(4):981-6. https://doi.org/10.1086/318116 PMid:11049780 
4. Wiersinga WJ, van der Poll T, White NJ, Day NP, Peacock SJ. Melioidosis: Insights into the pathogenicity of Burkholderia pseudomallei. Nat Rev Microbiol. 2006;4:272-82. https://doi. org/10.1038/nrmicro1385

5. Liang CC, Chen SY, Chen TY, Chen ST. Central nervous system melioidosis mimics malignancy: A case report and literature review. World Neurosurg. 2016;89:732.e19-23. https://doi. org/10.1016/j.wneu.2016.01.093

\section{PMid:26882971}

6. White ME, Hunt J, Connell C, Langdon K. Paediatric neurological melioidosis: A rehabilitation case report. Rural Remote Health. 2016;16(1):3702. https://doi.org/10.22605/rrh3702

PMid:26897103

7. Ng CS, Azmin S, Law ZK, Sahathevan R, Wan Yahya WN Remli R, et al. An unusual neurological complication from a garden-variety organism: post-melioidosis parkinsonism. Med J Aust. 2015;202(6):333-4. https://doi.org/10.5694/mja14.00229 PMid:25832163

8. Wijekoon P, Bandara KA, Kailainathan A, Chandrasiri NS, Hapuarachchi CT. Guillaine-barre syndrome: A rare complication of melioidosis. A case report. BMC Infect Dis. 2016;16:388. https://doi.org/10.1186/s12879-016-1719-4

PMid:27506202

9. Chen GB, Tuan SH, Chen LH, Lin WS. Neurological melioidosis (Burkholderia pseudomallei) in a chronic psychotic patient treated with antipsychotics: A case report. Medicine (United States). 2018;97(24):e11110. https://doi.org/10.1097/ MD.0000000000011110

PMid:29901631

10. Kumar MP, Krishnamurthy S, Venkateswaran VS, Mahadevan S, Lalitha M, Sistla S, et al. Brainstem micro-abscesses caused by Burkholderia pseudomallei in a 10-month-old infant: A case report. Paediatr Int Child Health. 2017;37(3):230-2. https://doi. org/10.1080/20469047.2016.1198560

PMid:27376438

11. Arif MA, Abid MH, Renganathan R, Siddiqui KA. Central and peripheral nervous system involvement in neuromelioidosis. BMJ Case Rep. 2015;2015:bcr2015211001. https://doi. org/10.1136/bcr-2015-211001 PMid:26494715

12. Loh TL, Latis S, Crossland G, Patel H. Disseminated melioidosis in the head and neck. BMJ Case Rep. 2017;2017:bcr2016218606. https://doi.org/10.1136/bcr-2016-218606

PMid:28096229

13. Chia-Tsong Hsu C, Singh D, Kwan G, Deuble M, Aquilina C, Korah I, et al. Neuromelioidosis: Craniospinal MRI findings in Burkholderia pseudomallei infection. J Neuroimaging. 2016;26:75-82. https://doi.org/10.1111/jon.12282 PMid:26256582

14. Huang WY, Wu G, Chen F, Li MM, Li JJ. Multi-systemic melioidosis: A clinical, neurological, and radiological case study from Hainan Province, China. BMC Infect Dis. 2018;18:649. https://doi.org/10.1186/s12879-018-3569-8

15. Amarasena HL, Silva FH, Tilakaratna PM, Jayamanne SF, Ranawaka UK. Melioidosis with a subdural collection a case report. BMC Infect Dis. 2019;19(1):143. https://doi.org/10.1186/ s12879-019-3782-0

PMid:30755178

16. Zhan $Y$, Wu Y, Li Q, Yu A. Neuromelioidosis: A series of seven cases in Hainan province, China. J Int Med Res. 2017;45(2):856-67. https://doi.org/10.1177/0300060516685967 PMid:28351287

17. Ganapathy S, Nair VK. Neuroinfectious diseases rare presentations of CNS melioidosis an institutional experience. J Neuroinfect Dis. 2018;9:2-5. https://doi. org/10.4172/2314-7326.1000278

18. Saravu K, Kadavigere R, Shastry AB, Pai R, Mukhopadhyay C Neurologic melioidosis presented as encephalomyelitis and subdural collection in two male labourers in India. J Infect Dev Ctries. 2015;9(11):1289-93. https://doi.org/10.3855/jidc.6586 PMid:26623640

19. Fong SL, Shyanwong J, Tan AH, Low SC, Tan CT. Neurological melioidosis in East Malaysia: Case series and review of the literature. Neurol Asia 2017;22(1):25-32.

20. Nayak R, Patel B, Raju K. Chronic pachymenigitis with dura venous sinus thrombosis: An unusual presentation of cranial melioidosis. Neurol India. 2018;66(4):1185-7. https://doi. org/10.4103/0028-3886.237015

PMid:30038121

21. Wiersinga WJ, Virk HS, Torres AG, Currie BJ, Peacock SJ, Dance DA, et al. Melioidosis. Nat Rev Dis Prim. 2018;4:17107. https://doi.org/10.1038/nrdp.2017.107

PMid:29388572

22. Limmathurotsakul D, Golding N, Dance DA, Messina JP, Pigott DM, Moyes CL, et al. Predicted global distribution of Burkholderia pseudomallei and burden of melioidosis. Nat Microbiol. 2016;1(1):15008. https://doi.org/10.1038/ nmicrobiol.2015.8

PMid:26877885

23. Currie BJ, Fisher DA, Anstey NM, Jacups SP. Melioidosis: Acute and chronic disease, relapse and re-activation. Trans $R$ Soc Trop Med Hyg. 2000;94(3):301-4. https://doi.org/10.1016/ s0035-9203(00)90333-x

PMid:10975006

24. Owen W, Smith S, Kuruvath S, Anderson D, Hanson J. Melioidosis of the central nervous system; a potentially lethal impersonator. IDCases. 2021;23(6):e01015. https://doi. org/10.1016/J.IDCR.2020.E01015

25. Lau SK, Sridhar S, Ho CC, Chow WN, Lee KC, Lam CW, et al. Laboratory diagnosis of melioidosis: Past, present and future. Exp Biol Med. 2015;240(6):742-51. https://doi. org/10.1177/1535370215583801 PMid:25908634

26. Currie BJ. Melioidosis: Evolving concepts in epidemiology, pathogenesis, and treatment. Semin Respir Crit Care Med. 2015;36(1):111-25. https://doi.org/10.1055/s-0034-1398389 PMid:25643275

27. Deuble $M$, Aquilina $C$, Norton R. Neurologic melioidosis. Am J Trop Med Hyg. 2013;89(3):535-9. https://doi.org/10.4269/ ajtmh.12-0559

PMid:23836574

28. Donahue MA, Newcomb G, Spinella S, Prasad P, Liesveld J, Egan CT, et al. CNS melioidosis in a traveler returning from cabo, Mexico. In: Open forum Infect. Oxford: Oxford University Press US; 2019. p. ofz005. https://doi.org/10.1093/ ofid/ofz005

29. Fong SL, Wong JS, Tan AH, Low SC, Tan CT. Neurologica melioidosis in East Malaysia: Case series and review of the literature. Neurol. Asia. 2017;22:25-32.

30. St John JA. Trojan horse L-selectin monocytes: A portal of Burkholderia pseudomallei entry into the brain. Virulence. 2017;8(6):611-2. https://doi.org/101080/2150559420161250997 PMid:27763813

31. St John JA, Walkden $H$, Nazareth L, Beagley KW, Ulett GC, Batzloff $M R$, et al. Burkholderia pseudomallei rapidly infects the brain stem and spinal cord via the trigeminal nerve after intranasal inoculation. Infect Immun. 2016;84(9):2681-8. https:// doi.org/10.1128/iai.00361-16 
PMid:27382023

32. Currie BJ, Ward L, Cheng AC. The epidemiology and clinical spectrum of melioidosis: 540 cases from the 20 year darwin prospective study. PLoS Negl Trop Dis. 2010;4(11):e900. https://doi.org/10.1371/journal.pntd.0000900

PMid:21152057

\section{Supplementary Table}

\section{Supplementary File 1}

\begin{tabular}{|c|c|c|c|}
\hline No & Authors & Title & Country \\
\hline 1 & Liang et al., 2016 [5] & $\begin{array}{l}\text { CNS Melioidosis mimics malignancy: A } \\
\text { case report and literature review }\end{array}$ & Taiwan \\
\hline 2 & White et al., 2016 [6] & $\begin{array}{l}\text { Pediatric neurological melioidosis: A } \\
\text { rehabilitation case report }\end{array}$ & Australia \\
\hline 3 & $\mathrm{Ng}$ et al., 2015 [7] & $\begin{array}{l}\text { An unusual neurological complication } \\
\text { from a garden-variety organism: post- } \\
\text { melioidosis parkinsonism }\end{array}$ & Malaysia \\
\hline 4 & Wijekoon et al., 2016 [8] & $\begin{array}{l}\text { Guillaine-barre syndrome; a rare } \\
\text { complication of melioidosis. a case report }\end{array}$ & Sri Lanka \\
\hline 5 & Chen et al., 2018 [9] & $\begin{array}{l}\text { Neurological melioidosis (Burkholderia } \\
\text { pseudomallei) in a chronic psychotic } \\
\text { patient treated with antipsychotics }\end{array}$ & Taiwan \\
\hline 6 & Kumar et al., 2017 [10] & $\begin{array}{l}\text { Brainstem micro-abscesses caused by } \\
\text { Burkholderia pseudomallei in a 10-month- } \\
\text { old infant: a case report }\end{array}$ & India \\
\hline 7 & Arif et al., 2015 [11] & $\begin{array}{l}\text { Central and peripheral nervous system } \\
\text { involvement in neuromelioidosis }\end{array}$ & Sri Lanka \\
\hline 8 & Loh et al., 2017 [12] & $\begin{array}{l}\text { Disseminated melioidosis in the head } \\
\text { and neck }\end{array}$ & Australia \\
\hline 9 & Hsu et al., 2016 [13] & $\begin{array}{l}\text { Neuromelioidosis: Craniospinal MRI } \\
\text { Findings in Burkholderia pseudomallei } \\
\text { Infection }\end{array}$ & Australia \\
\hline 10 & Huang et al., 2018 [14] & $\begin{array}{l}\text { Multi-systemic melioidosis: a clinical, } \\
\text { neurological, and radiological case study } \\
\text { from Hainan Province, China }\end{array}$ & China \\
\hline 11 & Amarasena et al., 2019 [15] & $\begin{array}{l}\text { Melioidosis with a subdural collection - a } \\
\text { case report }\end{array}$ & Sri Lanka \\
\hline 12 & Zhan et al., 2017 [16] & $\begin{array}{l}\text { Neuromelioidosis: a series of seven cases } \\
\text { in Hainan province, China }\end{array}$ & China \\
\hline 13 & Ganapathy et al., 2018 [17] & $\begin{array}{l}\text { Rare Presentations of CNS Melioidosis - } \\
\text { An Institutional Experience }\end{array}$ & India \\
\hline 14 & Saravu et al., 2015 [18] & $\begin{array}{l}\text { Neurologic melioidosis presented as } \\
\text { encephalomyelitis and subdural collection } \\
\text { in two male laborers in India }\end{array}$ & India \\
\hline 15 & Fong et al., 2017 [19] & $\begin{array}{l}\text { Neurological melioidosis in East Malaysia: } \\
\text { Case series and review of the literature }\end{array}$ & Malaysia \\
\hline 16 & Nayak et al., 2018 [20] & $\begin{array}{l}\text { Chronic pachymeningitis with dural venous } \\
\text { sinus thrombosis: An unusual presentation } \\
\text { of cranial melioidosis }\end{array}$ & India \\
\hline
\end{tabular}

